\title{
Comparison of Weed Suppression and Mandarin Fruit Yield and Quality Obtained with Organic Mulches, Synthetic Mulches, Cultivation, and Glyphosate
}

\author{
H.F. Abouziena ${ }^{1}$ \\ Botany Department, National Research Center, Dokki, Giza, Egypt
}

O.M. Hafez

Pomology Research Department, National Research Center, Dokki, Giza, Egypt

\author{
I.M. El-Metwally \\ Botany Department, National Research Center, Dokki, Giza, Egypt
}

S.D. Sharma and M. Singh

Horticultural Sciences Department, University of Florida, Institute of Food and Agricultural Sciences, Citrus Research and Education Center, 700 Experiment Road, Lake Alfred, FL 33850-2299

Additional index words. rice straw, cattail weed, wild oat mulch, weed control, plant mulch, plastic mulch, mulch deep, chemical properties

\begin{abstract}
Organic crop production, whether for export or local consumption, is increasing to avoid the residual effects of synthetic herbicides in foods, soil, and water, toxicity to other nontarget organisms, and herbicide-resistant weed populations. Organic farmers consistently ranked weed management as one of their most important production problems. Therefore, a 2-year study was conducted under 15-year-old mandarin trees to compare the effects of rice straw mulch, cattail mulch, black plastic mulch, hand hoeing, cultivation, glyphosate, and unweeded control treatments on weed control, fruit yield, and fruit quality. The greatest control $(94 \%-100 \%)$ of weeds occurred with the plastic mulch $(200$ or $150 \mu \mathrm{m})$ and three mulch layers of rice straw or cattail. Covering soil with cattail or rice straw mulch (two layers) gave $85 \%$ to $98 \%$ control of weeds. Uncontrolled weeds in the weedy control caused significant reduction in yield and fruit quality and decreased the yield/tree by $62 \%$ compared with hand hoe treatment. Plastic mulches of 200 and $150 \mu \mathrm{m}$, cattail (Cyprus articulatus L.) mulch (two or three layers) and two mulch layers of rice (Oryza sativa L.) straw treatments significantly increased the fruit yield/tree by $24 \%, 18 \%, 20 \%, 11 \%$, and $12 \%$ more than cultivation treatment, respectively, without significant differences among these superior treatments. Soil mulching with three layers of rice straw, cultivation, glyphosate, and $80-\mu \mathrm{m}$ plastic mulch treatments caused a significant reduction in weed density and weed biomass, but gave lower yield than superior treatments. Total soluble solids of fruits was unaffected by any of the weed management strategies, whereas values of total acidity and vitamin $C$ were significantly lower in the unwedded control than most weeded treatments. These results demonstrate that two layers of cattail or rice straw mulch could be used effectively for controlling weeds in citrus groves. Their effectiveness in controlling weeds may increase their use in agriculture systems with a concomitant decrease in the need for synthetic herbicides. Further studies are needed to evaluate their side effects on beneficial organisms, diseases, and insects.
\end{abstract}

Egyptian citrus, especially organic fruits, is exported to many countries. Weeds compete with citrus for nutrients and water, they harbor pests and host pathogens, and they lower the efficiency of orchard operations

Received for publication 17 Aug. 2007. Accepted for publication 29 Oct. 2007.

${ }^{1}$ To whom reprint requests should be addressed; e-mail abouzainah@yahoo.com
(Tucker and Singh, 1984). Chemical control of weeds in citrus was reported to affect various physical and chemical properties and quality of fruits (Mohanty et al., 2002; Sabbah et al., 1994).

In sandy soil with surface irrigation, weed infestation is much higher than with other types of irrigation. Manual weed control is temporary and significantly drains the resources of the growers. Indeed, Bredell (1973) reported that hand cultivation was not effec- tive in controlling weeds, as weed regrowth occurred rapidly after hand cultivation. Glyphosate has been the most widely used herbicide for postemergence weed control in citrus (Barbora et al., 2002; Martini et al., 2002; Sabbah et al., 1994; Singh and Singh, 2004). They also reported that overreliance on a single herbicide could result in loss of effectiveness from selection pressure on weeds, and there was evidence for a shift in weed flora. Weed species that are not effectively controlled by glyphosate have increased in citrus groves (Singh and Singh, 2004).

Interest in nonchemical weed control methods has been increasingly popular in recent years with the spread of organic farming and environmental concerns over adverse effects of herbicides. Radosevich et al. (1997) and Li et al. (2003), reported that the potential problems associated with herbicides use are injury to nontarget vegetation, crop injury, residues in soil and water, i.e., reduction of soil and water quality, toxicity to other nontarget organisms, concerns for human health and safety, and herbicide-resistant weed populations. Economically and environmentally sustainable weed control alternatives, such as nonsynthetic or natural mulch, can provide many benefits, including weed suppression and delayed weed seedling emergence (Bond and Grundy, 2001; Hussein and Radwan, 2002; Teasdale and Mohler, 1993), soil moisture conservation, and improved water infiltration (Faber et al., 2001; Hoyt and Hargrove, 1986). Benefits also include enhanced soil stabilization, soil porosity, water-holding capacity, microbial population activity, cation exchange capacity (Abdul-Baki and Teasdale, 1993), and decreased plant disease (Gleason et al., 2001). Synthetic mulches, manufactured from petroleum-based materials, have been used extensively in agriculture, but problems with these materials include increased runoff compared with living mulches, disposal and landfill concerns, and their restriction in "certified organic" production as a long-term management strategy. Consumption of organic products is increasing, and there is very little research support for this expanding production system (Doug et al., 2002).

Waste materials such as rice straw, weeds, aquatic weeds, bark, and composted municipal green waste can provide effective weed control. Because of a higher depth of mulch required to suppress weed emergence, it is likely that transport costs will be high, which may prohibit their use unless the material is produced on the farm (Merwin, 1995; Radwan and Hussein, 2001). The fresh bark of conifers, oak (Quercus spp.), and rape (Brassica napus L.) straw gave good control of weeds when they were used as mulches under apple (Malus domestica Borkh.) trees (Weibel and Niggli, 1990). Following straw mulch, fruit size and potassium content in the apple leaves increased with increasing organic mulches (Pedersen, 1999). However, organic mulches, especially wood chips, 
were ineffective in controlling perennial weeds, whereas the most effective was plastic mulch. However, Merwin (1995) reported that an alternative weed control strategy that provides potential benefits is mulching, although cost of mulching can be much higher than herbicides. Radwan and Hussein (2001) concluded that organic mulches, i.e., rice straw, sawdust, clover (Trifolium repens) weed, and cogon grass [Imperata cylindrica (L.) Palisot] significantly reduced the total dry weight of weeds in onion (Allium cepa $\mathrm{L}$.) fields. The dry weights of lambsquarters (Chenopodium album) and Italian ryegrass [Lolium perenne L. ssp. multiflorum (Lam.) Husnot] weeds increased with the use of sawdust as mulch. Broadleaf weeds were more susceptible to mulching than grassy weeds. Mukhopadhyay (2006) reported that the crop residue mulch with an allelopathic effects may be the most promising weed management practice in the future.

In Egypt, rice straw is considered one of the most important plant waste problems, and cattail weed is a problem in all water canals. The use of these materials as mulch may serve to eliminate the waste problem while providing weed control.

The objective of this study was to investigate the effect of plant mulches with different depths as physical methods in comparison with synthetic mulch, hand hoeing, cultivation, and glyphosate on weed control efficacy, and quantity and quality of mandarin fruits.

\section{Materials and Methods}

The study was conducted during 20052006 and 2006-2007 in a uniform 15-yearold Balady mandarin (Citrus reticulata Blanco) orchard budded on Sour orange (Citrus aurantium L.) in a private orchard located at El-Salhia District, Sharkia Governorate, Egypt. Trees were spaced $5 \times 5 \mathrm{~m}$ apart and were grown in a sandy soil using flood irrigation. Fertilization, irrigation, and other agricultural practices were applied as recommended. The soil texture of the experimental site was sandy with $1.41 \%$ organic matter, pH 7.9, E.C. $1.08 \mathrm{dsm}^{-1}$, and $1.78 \%$ $\mathrm{CaCO}_{3} ; 0.06 \mathrm{~N}, 0.017 \mathrm{P}, 0.02 \% \mathrm{~K}, 0.62 \mathrm{Zn}$, $0.4 \mathrm{Mn}$, and $3.7 \mathrm{Fe}$ ppm. The treatments presented in Table 1 were evaluated and compared for their effect on weed control, and quantity and quality of mandarin fruits.

Weeds at the base of the trees were controlled by hand. Treatments 4 through 13 were used as soil covers between the trees. Treatments were applied to six trees per plot and were replicated four times each year using randomized complete block design. Mulches were applied to plots during the first week of March each year, after winter cultivation, application of farmyard compost, and the first application of chemical fertilizer in the third week of February.

Weeds. The plots were visually rated for percentage of weed control 3, 5, 7, and 9 months after the mulch treatments application. We used a rating scale of 0 (no effect on weeds) to 100 (complete weed control) as approved by the Weed Science Society of America (Frans et al., 1986). Percentage control values were transformed to arcsine before analysis to improve the homogeneity of the error variance (Table 2). The original percentage scale is used in the tables. Weed counts were recorded per square meter randomly in each plot 3 and 6 months after treatment application in both years. Individual weeds were identified. Weeds were dried and their dry weights were determined.

Yield and yield components of mandarin trees. At harvest (mid-January) in both years, numbers of fruit per tree, fruit weight, and yield/tree were recorded.

Fruit quality characteristics. Samples of 20 fruit from each tree were randomly taken to determine the physical characters (fruit length, fruit diameter, and fruit length/diameter ratio). Chemical characteristics of fruit juice, i.e., total soluble solids, total acidity, ascorbic acid, and vitamin $\mathrm{C}$ were analyzed as described by the Association of Official Agriculture Chemists (1995).

Statistical analysis. The data from replicated experiments were combined, and after analysis showed nonsignificant year by treat- ment interactions. Analysis of variance was determined and the means were compared by Duncan's multiple-range test at 5\% level of significance (SAS, Cary, NC).

\section{Results and Discussion}

Effect of treatments on weeds. The major grass weeds present on the experimental site were bermudagrass [Cynodon dactylon (L.) Pers.], barnyardgrass [Echinochloa colonum (L.) Link], torpedo grass (Panicum repens L.), and sandbur (Cenchrus pennisetiformis, Hochst. \& Steud.) as a grass, and redroot pigweed (Amaranthus retroflexus L.), common purslane (Portulaca oleracea L.), and petty spurge (Euphorbia peplus L.) as broadleaf weeds. The dry weight of broadleaf weed species was less than the grass species as shown in unweeded treatment (Table 3 ). Maximum biomass was recorded from bermudagrass.

As shown in Tables 2 and 3, all weed control treatments were effective in reducing the weed density and dry weight compared with weedy control plots. Covering the soil of mandarin orchards with one layer of rice straw, cattail, and wild oat mulch, as well as the thinnest black plastic layer, had less effect against grasses, where torpedo grass emerged through the mulch. Similar results were reported by Mohanty et al. (2002) and Shirgure et al. (2003) in citrus orchards, and by Ghosh et al. (2006) in peanut fields. Radwan and Hussein (2001) reported that broadleaf weeds were more susceptible to mulching treatments than grassy weeds. Ligneau and Watt (1995) showed that a 3-cm layer of mulch was enough to prevent the emergence of annual weeds. The application of glyphosate proved the most efficient and maintained broadleaf and grass weed control up to 3 months (Table 3). The highest efficacy control of broad leaf weed $(94 \%$ reduction in dry weight) was observed after 4 months from glyphosate treatment compared with unweeded control treatment, where regrowth of grass had started. Variable glyphosate efficacy has been reported on different weed

Table 1 . Weed control treatments used in the 2-year experiment.

\begin{tabular}{|c|c|c|}
\hline Treatments & Abbreviations & Application \\
\hline 1. Unweeded control & UW & Allowing weeds to grow with mandarin trees \\
\hline 3. Cultivation & $\mathrm{Cul}$ & $\begin{array}{l}\text { Orchard floor was disked in two directions (east-west and north-south) with } \\
\text { a standard orchard disk twice annually (second week of May and August) }\end{array}$ \\
\hline 4. One layer of rice straw (Oryza sativa $\mathrm{L}$.) mulch & 1LRSM & $3 \mathrm{~cm}$ deep; 8 tons $/$ ha \\
\hline 5. Two layers of rice straw mulch & 2LRSM & $6 \mathrm{~cm}$ deep; 15 tons $/ \mathrm{ha}$ \\
\hline 6. Three layers of rice straw mulch & 3LRSM & $9 \mathrm{~cm}$ deep; 23 tons $/ \mathrm{ha}$ \\
\hline 8. Two layers of cattail weed mulch & 2LCWM & $8 \mathrm{~cm}$ deep; 20 tons $/ \mathrm{ha}$ \\
\hline 9. Three layers of cattail weed mulch & 3LCWM & $12 \mathrm{~cm}$ deep; 30 tons $/ \mathrm{ha}$ \\
\hline 10. Wild oat (Avena fatua, L.) mulch & WOM & $6 \mathrm{~cm}$ deep; 15 tons $/ \mathrm{ha}$ \\
\hline 11. Black plastic mulch & $80 \mu \mathrm{m}$ BPM. & $80 \mu \mathrm{m}$ thickness \\
\hline 12. Black plastic mulch & $150 \mu \mathrm{m}$ BPM & $150 \mu \mathrm{m}$ thickness \\
\hline 13. Black plastic mulch & $200 \mu \mathrm{m}$ BPM & $200 \mu \mathrm{m}$ thickness \\
\hline 14. Glyphosate herbicide & Gly. & $\begin{array}{l}\text { Glyphosate (Roundup) at } 850 \mathrm{~g} \text { a.i/ha was applied to the orchard floor in the } \\
\text { second week of May. Source of Roundup, Monsanto Company, } 800 \\
\text { North Lindbergh, Boulevard, St. Louis, MO } 63167 \text {. }\end{array}$ \\
\hline
\end{tabular}


Table 2. Influence of organic and synthetic mulches, hand hoeing, cultivation, and glyphosate on weed control in mandarin orchard.

\begin{tabular}{|c|c|c|c|c|}
\hline \multirow[b]{2}{*}{ Treatments ${ }^{z}$} & \multicolumn{4}{|c|}{ Weed control $\%{ }^{y}$} \\
\hline & June 10 & Aug. 10 & Oct.10 & Dec.10 \\
\hline$\overline{\mathrm{UW}}$ & $25 \mathrm{e}^{\mathrm{x}}$ & $17 \mathrm{f}$ & $9 \mathrm{f}$ & $11 \mathrm{~g}$ \\
\hline $\mathrm{HH}$ & $96 \mathrm{~b}$ & $78 \mathrm{c}$ & $63 \mathrm{~d}$ & $49 \mathrm{e}$ \\
\hline Cul & $89 \mathrm{c}$ & $67 \mathrm{~d}$ & $52 \mathrm{e}$ & $37 \mathrm{f}$ \\
\hline 1LRSM & $73 \mathrm{~d}$ & $68 \mathrm{~d}$ & $58 \mathrm{ed}$ & $38 \mathrm{f}$ \\
\hline 2LRSM & $98 \mathrm{ab}$ & $92 \mathrm{~b}$ & $91 \mathrm{~b}$ & 89 bc \\
\hline 3LRSM & $100 \mathrm{a}$ & $99 \mathrm{a}$ & $97 \mathrm{ab}$ & $94 \mathrm{ab}$ \\
\hline 1LCWM & $74 \mathrm{~d}$ & $66 \mathrm{~d}$ & 55 ed & $49 \mathrm{e}$ \\
\hline 2LCWM & $96 \mathrm{~b}$ & $95 \mathrm{ab}$ & $90 \mathrm{~b}$ & $85 \mathrm{c}$ \\
\hline 3LCWM & $100 \mathrm{a}$ & $98 \mathrm{a}$ & $97 \mathrm{ab}$ & $95 \mathrm{ab}$ \\
\hline WOM & $74 \mathrm{~d}$ & $53 \mathrm{e}$ & $53 \mathrm{e}$ & 42 ef \\
\hline $80 \mu \mathrm{m}$ BPM & $100 \mathrm{a}$ & $96 \mathrm{ab}$ & $74 \mathrm{c}$ & $69 \mathrm{~d}$ \\
\hline $150 \mu \mathrm{m}$ BPM & $100 \mathrm{a}$ & $100 \mathrm{a}$ & $100 \mathrm{a}$ & $98 \mathrm{a}$ \\
\hline $200 \mu \mathrm{m}$ BPM & $100 \mathrm{a}$ & $100 \mathrm{a}$ & $100 \mathrm{a}$ & $100 \mathrm{a}$ \\
\hline Gly. & $92 \mathrm{c}$ & $76 \mathrm{c}$ & 56 ed & $41 \mathrm{f}$ \\
\hline $\mathrm{CV} \%$ & 2.4 & 5 & 17.3 & 4.6 \\
\hline
\end{tabular}

${ }^{\mathrm{z} U W:}$ unweeded, HH: hand hoeing, Cul: cultivation, LRSM: layer of rice straw mulch, LCWM: layer of cattail weed mulch, WOM: wild oat mulch, BPM: black plastic mulch, Gly: glyphosate.

${ }^{\mathrm{y}}$ Rating scale $(0=$ no effect and $100=$ complete control).

${ }^{\mathrm{x}}$ Treatment means within a column followed by the same letter do not differ significantly $(P=0.05$, Student-Newman-Keuls) probability.

species (Singh and Singh, 2003; Wehtje and Walker, 1997).

Among plant mulches, two or three layers of cattail mulch resulted in the lowest density
(Table 2) and dry weight (Table 3 ) of weeds followed by rice straw mulch. Covering the soil with cattail mulch at 2 and 3 layers caused a significant reduction in dry weight of weeds up to 6 months after application by $95 \%$ and $99 \%$, respectively, compared with unweeded control (Tables 3). Similar to cattail mulch, the application of rice straw mulch at the same layers provided similar control. This efficacy may be because of light interception and allelopathy (Daar, 1986; Mohanty et al., 2002). Abou Sayed-Ahmed et al. (2005) also found that mulch treatments resulted in the most effective control of broadleaf, grassy, and perennial weeds of oranges. Our data support previous studies that organic mulches provide effective weed control if applied at a sufficient depth (Mohanty et al., 2002; Robinson, 1988; Singh et al., 1985). Hand hoeing and cultivation gave $96 \%$ and $89 \%$ weed control, respectively, for up to 2 months (Table 2), after which, the weeds regrow, especially perennial weeds (Table 3). Donadio et al. (1988) and Ahmed and El-Bestawesy (1995) found a significant shift of weed species after hoeing in a citrus orchard, and Bredell (1973) mentioned that hand cultivation was not effective in controlling weeds and weed regrowth occurred rapidly.

Our results indicated that wild oat mulch resulted in $74 \%$ weed control for only 3 months (Table 2). Black plastic mulch of 150 or $200 \mu \mathrm{m}$ resulted in the highest weed control, which was on par with cattail or rice straw mulch at two layers. Mohanty et al. (2002) reported that a black polyethylene $(300 \mu \mathrm{m})$ sheet completely eliminated all weed species from nonpenetration of sunlight. Two layers of cattail or rice straw were better than hand hoeing, cultivation, and glyphosate (Tables 2 and 3). Abou SayedAhmed et al. (2005) reported that mulching with plastic or straw in a citrus orchard controlled weed growth to a considerable extent, depending on weed species. However, Timothy (2007) reported that organic mulch of any type greatly reduced dicot weed number with no type superior to the others. From the previous data, it could be concluded that two or three mulch layers of cattail mulch and rice straw mulch were effective for controlling weeds in mandarin orchards.

Effect of treatments on yield and fruit physical properties of mandarin fruit. As shown in Table 4, the application of black plastic mulch at $80 \mu \mathrm{m}$ increased the quantity and quality yield of mandarin trees by $119 \%$ compared with the unweeded plot. The quantity and quality under $80 \mu \mathrm{m}$ of plastic mulch were less than hand hoeing and cattail mulch (two layers).

Fruit length was unaffected by any of the weed control treatments, whereas other characteristics were significantly affected. Weeds growing with the mandarin trees in nonweeded control plots caused a significant reduction in fruit diameter, weight, and number

Table 3. Influence of organic and synthetic mulches, hand hoeing, cultivation, and glyphosate on the dry weight of weeds $\left(\mathrm{g} / \mathrm{m}^{2}\right)$ grown in mandarin orchards.

\begin{tabular}{|c|c|c|c|c|c|c|c|c|c|c|}
\hline \multirow[b]{2}{*}{ Treatments ${ }^{z}$} & \multicolumn{4}{|c|}{ Broadleaf weeds } & \multicolumn{5}{|c|}{ Grasses } & \multirow[b]{2}{*}{ Total } \\
\hline & $\begin{array}{l}\text { Redroot } \\
\text { pigweed }\end{array}$ & $\begin{array}{l}\text { Common } \\
\text { purslane }\end{array}$ & $\begin{array}{c}\text { Petty } \\
\text { spurge }\end{array}$ & Total & $\begin{array}{l}\text { Bermuda } \\
\text { grass }\end{array}$ & $\begin{array}{c}\text { Barnyard } \\
\text { grass }\end{array}$ & $\begin{array}{c}\text { Torpedo } \\
\text { grass }\end{array}$ & Sandbur & Total & \\
\hline \multicolumn{11}{|l|}{ June 12} \\
\hline UW & $30 \mathrm{~b}^{\mathrm{y}}$ & $27 \mathrm{~b}$ & $47 \mathrm{~d}$ & $104 \mathrm{c}$ & $105 \mathrm{c}$ & $39 \mathrm{c}$ & $23 d$ & $25 \mathrm{~b}$ & $192 d$ & $296 \mathrm{f}$ \\
\hline $\mathrm{HH}$ & $0 \mathrm{a}^{*}$ & $2 \mathrm{~b}$ & $0 \mathrm{a}$ & $2 \mathrm{a}$ & $2 \mathrm{a}$ & $1 \mathrm{ab}$ & $2 \mathrm{a}$ & $2 \mathrm{a}$ & $7 \mathrm{ab}$ & $9 \mathrm{abc}$ \\
\hline Cul & $1 \mathrm{a}$ & $3 \mathrm{~b}$ & $1 \mathrm{a}$ & $5 a$ & $4 \mathrm{a}$ & $3 a b$ & $2 a$ & $3 a$ & $12 \mathrm{ab}$ & $17 \mathrm{abc}$ \\
\hline 1LRSM & $0 \mathrm{a}$ & $2 \mathrm{~b}$ & $16 \mathrm{bc}$ & $18 \mathrm{~b}$ & $2 a$ & $16 \mathrm{ab}$ & $12 \mathrm{bc}$ & $10 \mathrm{a}$ & $40 \mathrm{bc}$ & $58 \mathrm{~cd}$ \\
\hline 2LRSM & $2 a$ & $3 \mathrm{~b}$ & $16 \mathrm{bc}$ & $21 \mathrm{~b}$ & $12 \mathrm{ab}$ & $5 \mathrm{ab}$ & $1 \mathrm{a}$ & $1 \mathrm{a}$ & $19 \mathrm{ab}$ & $40 \mathrm{bcd}$ \\
\hline 3LRSM & $1 \mathrm{a}$ & $2 \mathrm{~b}$ & $4 \mathrm{ab}$ & $7 \mathrm{a}$ & $0 \mathrm{a}$ & $2 a b$ & $1 \mathrm{a}$ & $1 \mathrm{a}$ & $4 \mathrm{ab}$ & $11 \mathrm{abc}$ \\
\hline 1LCWM & $2 a$ & $3 \mathrm{~b}$ & $16 \mathrm{bc}$ & $21 \mathrm{~b}$ & $16 \mathrm{ab}$ & $5 \mathrm{ab}$ & $2 a$ & $3 a$ & $26 \mathrm{ab}$ & $47 \mathrm{~cd}$ \\
\hline 2LCWM & $1 \mathrm{a}$ & $2 \mathrm{~b}$ & $3 a$ & $6 a$ & $1 \mathrm{a}$ & $0 \mathrm{a}$ & $0 \mathrm{a}$ & $0 \mathrm{a}$ & $1 \mathrm{a}$ & $7 \mathrm{abc}$ \\
\hline 3LCWM & $0 \mathrm{a}$ & $1 \mathrm{~b}$ & $3 a$ & $4 \mathrm{a}$ & $0 \mathrm{a}$ & $0 \mathrm{a}$ & $1 \mathrm{a}$ & $0 \mathrm{a}$ & $1 \mathrm{a}$ & $5 \mathrm{ab}$ \\
\hline WOM & $2 a$ & $3 \mathrm{~b}$ & $24 \mathrm{c}$ & $29 \mathrm{~b}$ & $23 \mathrm{~b}$ & $17 \mathrm{~b}$ & $5 \mathrm{a}$ & $24 \mathrm{~b}$ & $69 \mathrm{c}$ & $98 \mathrm{e}$ \\
\hline $80 \mu \mathrm{m}$ BPM & $1 \mathrm{a}$ & $0 \mathrm{~b}$ & $0 \mathrm{a}$ & $1 \mathrm{a}$ & $1 \mathrm{a}$ & $7 \mathrm{ab}$ & $15 \mathrm{c}$ & $0 \mathrm{a}$ & $23 \mathrm{ab}$ & $24 \mathrm{bcd}$ \\
\hline $150 \mu \mathrm{m}$ ВPM & $0 \mathrm{a}$ & $0 \mathrm{~b}$ & $0 \mathrm{a}$ & $0 \mathrm{a}$ & $1 \mathrm{a}$ & $0 \mathrm{a}$ & $6 \mathrm{bc}$ & $0 \mathrm{a}$ & $7 \mathrm{ab}$ & $7 \mathrm{abc}$ \\
\hline $200 \mu \mathrm{m}$ BPM & $0 \mathrm{a}$ & $0 \mathrm{~b}$ & $0 \mathrm{a}$ & $0 \mathrm{a}$ & $0 \mathrm{a}$ & $0 \mathrm{a}$ & $0 \mathrm{a}$ & $0 \mathrm{a}$ & $0 \mathrm{a}$ & $0 \mathrm{a}$ \\
\hline Gly. & $0 \mathrm{a}$ & $1 \mathrm{a}$ & $2 \mathrm{a}$ & $3 a$ & $4 a b$ & $1 \mathrm{a}$ & $3 a$ & $0 \mathrm{a}$ & $8 \mathrm{ab}$ & $11 \mathrm{abc}$ \\
\hline $\mathrm{CV} \%$ & 3.1 & 3.7 & 7.4 & 6.7 & 12.3 & 9.6 & 4.0 & 6.5 & 23.5 & 23.8 \\
\hline \multicolumn{11}{|l|}{ Sept. 1} \\
\hline UW & $91 \mathrm{c}$ & $25 \mathrm{c}$ & $175 \mathrm{c}$ & $317 \mathrm{~d}$ & $207 \mathrm{~b}$ & $95 \mathrm{~b}$ & $119 \mathrm{~d}$ & $26 \mathrm{~d}$ & $448 \mathrm{~d}$ & $765 \mathrm{~g}$ \\
\hline $\mathrm{HH}$ & $7 \mathrm{ab}$ & $7 \mathrm{a}$ & $3 \mathrm{a}$ & $11 \mathrm{a}$ & $15 \mathrm{a}$ & $10 \mathrm{a}$ & $15 \mathrm{ab}$ & $1 \mathrm{a}$ & $40 \mathrm{ab}$ & $52 \mathrm{ab}$ \\
\hline Cul & $8 a b$ & $7 \mathrm{a}$ & $6 a$ & $20 \mathrm{a}$ & $17 \mathrm{a}$ & $13 \mathrm{a}$ & $23 \mathrm{ab}$ & $8 \mathrm{~b}$ & $66 \mathrm{ab}$ & $81 \mathrm{bcd}$ \\
\hline 1LRSM & $5 \mathrm{a}$ & $43 \mathrm{c}$ & $7 \mathrm{a}$ & $57 \mathrm{c}$ & $26 a$ & $38 \mathrm{a}$ & $39 \mathrm{~b}$ & $17 \mathrm{c}$ & $120 \mathrm{~b}$ & $178 \mathrm{e}$ \\
\hline 2LRSM & $0 \mathrm{a}$ & $1 \mathrm{a}$ & $3 a$ & $4 a$ & $9 \mathrm{a}$ & $9 \mathrm{a}$ & $6 a b$ & $3 a b$ & $26 \mathrm{a}$ & $30 \mathrm{ab}$ \\
\hline 3LRSM & $0 \mathrm{a}$ & $4 a$ & $0 \mathrm{a}$ & $4 \mathrm{a}$ & $6 a$ & $0 \mathrm{a}$ & $6 a b$ & $0 \mathrm{a}$ & $12 \mathrm{a}$ & $18 \mathrm{a}$ \\
\hline 1LCWM & $30 \mathrm{~b}$ & $16 \mathrm{bc}$ & $10 \mathrm{a}$ & $56 \mathrm{bc}$ & $18 \mathrm{a}$ & $8 \mathrm{a}$ & $25 \mathrm{ab}$ & $7 \mathrm{ab}$ & $58 \mathrm{ab}$ & $114 \mathrm{~cd}$ \\
\hline 2LCWM & $5 \mathrm{a}$ & $5 \mathrm{a}$ & $15 \mathrm{a}$ & $24 \mathrm{ab}$ & $2 a$ & $2 a$ & $7 \mathrm{ab}$ & $1 \mathrm{a}$ & $12 \mathrm{a}$ & $36 a b$ \\
\hline 3LCWM & $0 \mathrm{a}$ & $2 \mathrm{a}$ & $0 \mathrm{a}$ & $2 a$ & $0 \mathrm{a}$ & $0 \mathrm{a}$ & $0 \mathrm{a}$ & $3 a b$ & $3 \mathrm{a}$ & $6 \mathrm{a}$ \\
\hline WOM & $15 \mathrm{ab}$ & $4 \mathrm{a}$ & $42 \mathrm{~b}$ & $61 \mathrm{c}$ & $20 \mathrm{a}$ & $96 \mathrm{~b}$ & $85 \mathrm{c}$ & $4 \mathrm{ab}$ & $205 \mathrm{c}$ & $265 \mathrm{f}$ \\
\hline $80 \mu \mathrm{m}$ BPM & $4 \mathrm{a}$ & $3 a$ & $7 \mathrm{a}$ & $14 \mathrm{a}$ & $6 a$ & $1 \mathrm{a}$ & $38 \mathrm{~b}$ & $1 \mathrm{a}$ & $46 \mathrm{ab}$ & $60 \mathrm{abc}$ \\
\hline $150 \mu \mathrm{m}$ ВРM & $0 \mathrm{a}$ & $0 \mathrm{a}$ & $0 \mathrm{a}$ & $0 \mathrm{a}$ & $0 \mathrm{a}$ & $0 \mathrm{a}$ & $0 \mathrm{a}$ & $0 \mathrm{a}$ & $0 \mathrm{a}$ & $0 \mathrm{a}$ \\
\hline $200 \mu \mathrm{m} \mathrm{BPM}$ & $0 \mathrm{a}$ & $0 \mathrm{a}$ & $0 \mathrm{a}$ & $0 \mathrm{a}$ & $0 \mathrm{a}$ & $0 \mathrm{a}$ & $0 \mathrm{a}$ & $0 \mathrm{a}$ & $0 \mathrm{a}$ & $0 \mathrm{a}$ \\
\hline Gly. & $6 a$ & $10 \mathrm{ab}$ & $2 a$ & $18 \mathrm{a}$ & $45 \mathrm{a}$ & $39 a$ & $27 \mathrm{ab}$ & $7 \mathrm{~b}$ & $117 \mathrm{~b}$ & $134 \mathrm{de}$ \\
\hline $\mathrm{CV} \%$ & 11.6 & 11.1 & 6.9 & 4.8 & 10 & 13.1 & 7.3 & 6.2 & 5.9 & 2.9 \\
\hline
\end{tabular}

${ }^{2}$ UW: unweeded, HH: hand hoeing, Cul: cultivation, LRSM: layer of rice straw mulch, LCWM: layer of cattail weed mulch, WOM: wild oat mulch, BPM: black plastic mulch, Gly: glyphosate; * 0: did not occur.

${ }^{\mathrm{y}}$ Treatment means within a column followed by the same letter do not differ significantly $(P=0.05$, Student-Newman-Keuls) probability. 
Table 4. Influence of organic and synthetic mulches, hand hoeing, cultivation, and glyphosate on yield components and fruit physical and chemical properties of mandarin fruits.

\begin{tabular}{|c|c|c|c|c|c|c|c|c|}
\hline Treatments $^{\mathrm{z}}$ & $\begin{array}{c}\text { Fruit } \\
\text { length }(\mathrm{cm})\end{array}$ & $\begin{array}{c}\text { Fruit } \\
\operatorname{diam}(\mathrm{cm})\end{array}$ & $\begin{array}{l}\text { Length/diameter } \\
\text { ratio }\end{array}$ & $\begin{array}{l}\text { Fruit } \\
\text { wt }(g)\end{array}$ & $\begin{array}{l}\text { No. of } \\
\text { fruit/tree }\end{array}$ & $\begin{array}{l}\text { Total soluble } \\
\text { solids }(\%)\end{array}$ & $\begin{array}{c}\text { Total } \\
\text { acidity }(\%)\end{array}$ & $\begin{array}{c}\text { V.C } \\
(\mathrm{mg} / 100 \mathrm{ml})\end{array}$ \\
\hline$\overline{\mathrm{UW}}$ & $5.5 \mathrm{~cd}^{\mathrm{y}}$ & $4.9 \mathrm{f}$ & $1.1 \mathrm{a}$ & $127 \mathrm{~g}$ & $255 \mathrm{f}$ & $10 \mathrm{a}$ & $0.7 \mathrm{~d}$ & $41 \mathrm{~d}$ \\
\hline $\mathrm{HH}$ & $5.8 \mathrm{cb}$ & $6.2 \mathrm{a}$ & $0.9 \mathrm{c}$ & $170 \mathrm{dc}$ & $494 \mathrm{abc}$ & $11 \mathrm{a}$ & $0.8 \mathrm{bcd}$ & $47 \mathrm{abcd}$ \\
\hline Cul & $5.6 \mathrm{~cd}$ & $6.1 \mathrm{a}$ & $0.9 \mathrm{c}$ & 166 dce & 448 bc & $12 \mathrm{a}$ & $1.0 \mathrm{abc}$ & $49 \mathrm{ab}$ \\
\hline 1LRSM & $5.1 \mathrm{f}$ & $5.7 \mathrm{cb}$ & $0.9 \mathrm{c}$ & $171 \mathrm{bc}$ & $357 \mathrm{ed}$ & $12 \mathrm{a}$ & $0.9 \mathrm{bcd}$ & $47 \mathrm{abcd}$ \\
\hline 2LRSM & $5.4 \mathrm{edf}$ & $6.2 \mathrm{a}$ & $0.9 \mathrm{c}$ & $169 \mathrm{dc}$ & $490 \mathrm{abc}$ & $11 \mathrm{a}$ & $0.8 \mathrm{~cd}$ & $50 \mathrm{ab}$ \\
\hline 3LRSM & $5.6 \mathrm{~cd}$ & $5.7 \mathrm{cb}$ & $1.0 \mathrm{~b}$ & $157 \mathrm{dfe}$ & $490 \mathrm{abc}$ & $12 \mathrm{a}$ & $1.1 \mathrm{ab}$ & $43 \mathrm{bcd}$ \\
\hline 1LCWM & $5.6 \mathrm{~cd}$ & $5.3 \mathrm{ed}$ & $1.1 \mathrm{a}$ & 154 ef & 322 ef & $11 \mathrm{a}$ & $1.0 \mathrm{abc}$ & $42 \mathrm{~cd}$ \\
\hline 2LCWM & $5.6 \mathrm{~cd}$ & $5.8 \mathrm{~b}$ & $1.0 \mathrm{~b}$ & 163 dce & $545 \mathrm{a}$ & $10 \mathrm{a}$ & $0.7 \mathrm{~cd}$ & 43 bcd \\
\hline 3LCWM & $5.5 \mathrm{ed}$ & $5.8 \mathrm{~b}$ & $1.0 \mathrm{~b}$ & 161 ecdf & $506 \mathrm{ab}$ & $11 \mathrm{a}$ & $0.8 \mathrm{bcd}$ & 46 abcd \\
\hline WOM & $4.8 \mathrm{~g}$ & $5.1 \mathrm{ef}$ & $0.9 \mathrm{c}$ & $148 \mathrm{f}$ & $341 \mathrm{e}$ & $12 \mathrm{a}$ & $1.3 \mathrm{a}$ & $49 \mathrm{ab}$ \\
\hline $80 \mu \mathrm{m}$ BPM & $6.3 \mathrm{a}$ & $6.2 \mathrm{a}$ & $1.0 \mathrm{~b}$ & $166 \mathrm{cde}$ & $424 \mathrm{~cd}$ & $11 \mathrm{a}$ & $0.7 \mathrm{~d}$ & $41 \mathrm{~d}$ \\
\hline $150 \mu \mathrm{m}$ BPM & $5.2 \mathrm{ef}$ & $5.5 \mathrm{~cd}$ & $0.9 \mathrm{c}$ & $184 \mathrm{ab}$ & $466 \mathrm{abc}$ & $10 \mathrm{a}$ & $0.7 \mathrm{~d}$ & $40 \mathrm{~d}$ \\
\hline $200 \mu \mathrm{m}$ BPM & $5.5 \mathrm{~cd}$ & $5.6 \mathrm{cb}$ & $1.0 \mathrm{~b}$ & $185 \mathrm{a}$ & $495 \mathrm{abc}$ & $10 \mathrm{a}$ & $0.9 \mathrm{bcd}$ & $49 \mathrm{ab}$ \\
\hline Gly. & $6.0 \mathrm{~b}$ & $5.7 \mathrm{cb}$ & $1.0 \mathrm{~b}$ & $162 \mathrm{ced}$ & $444 \mathrm{bc}$ & $11 \mathrm{a}$ & $0.8 \mathrm{~cd}$ & $51 \mathrm{a}$ \\
\hline $\mathrm{CV} \%$ & 0.2 & 0.2 & 0.1 & 8.1 & 48.4 & 1 & 0.2 & 4.2 \\
\hline
\end{tabular}

${ }^{\mathrm{z} U W}$ : unweeded, HH: hand hoeing, Cul: cultivation, LRSM: layer of rice straw mulch, LCWM: layer of cattail weed mulch, WOM: wild oat mulch, BPM: black plastic mulch, Gly: glyphosate; *0: did not occur.

${ }^{\mathrm{y}}$ Treatment means within a column followed by the same letter do not differ significantly $(P=0.05$, Student-Newman-Keuls $)$ probability.

of fruit per tree by $21 \%, 25 \%$, and $48 \%$, respectively, compared with hand hoeing (Table 4). Similar results were reported by Sabbah et al. (1994), Santinoni and Silva (1995), and Lima et al. (2002).

The reduction in fruit yield per tree from weeds was $62 \%$ (Fig. 1). This may be unjustified speculation because of the diffusion of toxic root exudates into the soil which affected mandarin tree growth (Aharoni et al., 1969). Kalita and Bhattacharyya (1995) reported that controlling weeds in lemon (Citrus limon Burm.f.) orchards improved flowering, fruiting, and fruit yield. MacRae et al. (2007) reported that interference from weeds with peach (Prunus persica) trees would reduced the availability of water and nutrients, and limited the amount of cell division thus affecting the final size of the fruit. These studies may explain the decrements of number, diameter, and weight of fruits in unweeded control treatment. Data in Table 4 also indicate that all weed control treatments had a significant increment in fruit criteria, in most cases, than unweeded control. Using one mulch layer of rice straw resulted in more yield than that of one layer of cattail mulch by $22 \%$ (Fig. 1). The application of two layers of rice straw resulted in more yield per tree by $8 \%$ than did three layers, whereas two layers of cattail gave $9 \%$ more than three layers. However, using three layers of mulch resulted in higher levels of weed control than two layers (Tables 2 and $3)$. We conclude that two layers of rice straw mulch $(6 \mathrm{~cm}$ deep) or cattail mulch $(8 \mathrm{~cm}$ deep) are sufficient to control weeds in mandarin groves. Advantages of effective control by these mulches include lower weed management costs, more yield per tree, and less competition for irrigation water and nutrients. Previous studies found a pro-

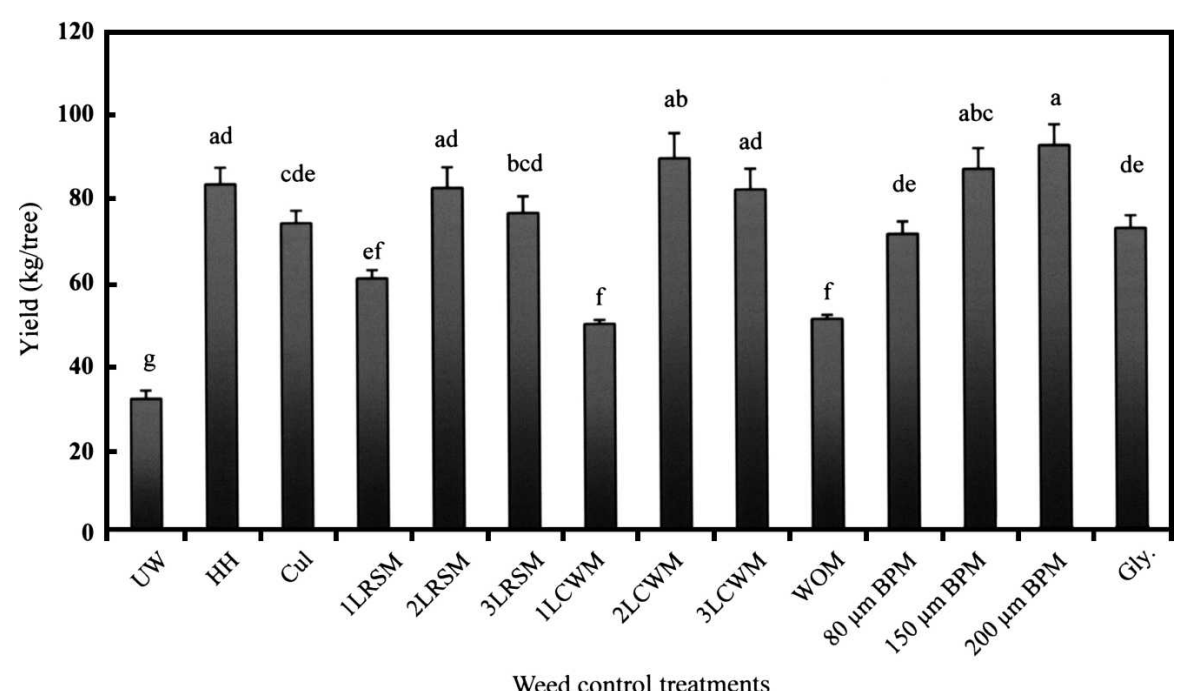

Fig. 1. Yield of mandarin trees as affected by organic or synthetic mulch, hand hoeing, cultivation, and glyphosate treatments. Bars labeled with the same letter are not significantly different $(P=0.05$, Student-Newman-Keuls) probability. UW: unweeded, HH: hand hoeing, Cul: cultivation, LRSM: layer of rice straw mulch, LCWM: layer of cattail weed mulch, WOM: wild oat mulch, BPM: black plastic mulch, Gly: glyphosate. nounced effect of mulch on soil moisture by (reduced evaporative loss) and weed growth (substantially reduced with mulch; Faber et al., 2001).

Using plastic mulches at 200 and $150 \mu \mathrm{m}$, two layers of cattail or rice straw mulch, and hand hoeing for controlling weeds resulted in the highest yield per tree without significant differences between these treatments. Soil mulching with rice straw or cattail at three layers, as well as mechanical hoeing, glyphosate, and plastic mulch $(80 \mu \mathrm{m})$ treatments caused a significant reduction in weed density and weed biomass, but gave a lower yield compared with superior treatments. This result may be because of a short period of effective control and less efficacy control on grass (Table 3 ) of these previous treatments. Tworkoski and Glenn (2001) reported that the grasses in a peach grove were one of the most competitive species and reduced vertical water sprout length by $15 \%$ to $27 \%$ and lateral shoot length on fruit-bearing branches by $19 \%$ to $30 \%$ compared with herbicide treatments. MacRae et al. (2007) reported that maintaining the orchard floor weed-free for 12 weeks after peach tree bloom resulted in the greatest fruit size (individual fruit weight and diameter), total yield, and fruit number.

Effect of treatments on fruit chemical properties of mandarin. Total soluble solids was unaffected by weed control treatments, whereas total acidity and vitamin $\mathrm{C}$ values were significantly influenced by weed control treatments (Table 4). Goff et al. (1991) reported that pecan leaf concentrations of $\mathrm{N}, \mathrm{P}, \mathrm{B}, \mathrm{Cu}$, and $\mathrm{Fe}$ were not significantly affected by weed control treatments. Also, Eissa and Helail (1997) found that rice straw mulching in Balady lime (Citrus aurantifolia) showed high efficiency in controlling weeds, enhancing transplant growth, and improving leaf content of phosphorus, potassium, magnesium, iron, and zinc. Timossi et al. (2001) reported that the clomazone herbicide had no effects on the Hamlin orange (Citrus sinensis) juice quality. Mohanty 
et al. (2002) reported that no significant difference in total soluble solids of mandarin fruits was observed among mulch treatments (black plastic mulch, soybean straw, local grasses, and paddy straw), but acidity was least with a black polyethylene sheet and was highest with unweeded treatment.

Generally, controlling weeds led to an increase in the fruit chemical properties of mandarin compared with the unweeded control.

\section{Conclusion}

Evaluation of mulching with rice straw, wild oat, and cattail weed for controlling weeds in mandarin orchards indicated that two layers of cattail or rice straw mulch could be used effectively for controlling weeds in citrus orchards. Their effectiveness in controlling weeds may increase their use in agriculture systems with a concomitant decrease in the need for synthetic herbicides. Further studies are needed to evaluate their side effects on beneficial organisms, diseases, and insects.

\section{Literature Cited}

Abdul-Baki, A.A. and J.R. Teasdale. 1993. A notillage tomato production system using hairy vetch and subterranean clover mulches. HortScience 28:106-108.

Abou Sayed-Ahmed, T.A., R.A. Al-Ashkar, L.A. El-Mashad, and A.R. Bdr El-Deen. 2005. Comparative study of some integrated weed control treatments on Washington navel orange trees and associated weeds. Zagazig J. Agr. Res. (Egypt) 32:35-56.

Aharoni, M., W. Hiller, and J. Patt. 1969. Effect of drainage water from the root medium of bermuda grass (Cynodon dactylon) and of annual weeds on the development of citrus seedlings. Israel J. Agr. Res. 19:74-78.

Ahmed, S.A. and F.I. El-Bestawesy. 1995. Effect of some herbicides used alone or in combination with urea on Navel orange trees and associated weeds. J. Agr. Sci. Mansoura Univ. (Egypt) 20:3181-3189.

Association of Official Agriculture Chemists. 1995. Association of Official Agriculture Chemists. Official methods of analysis. 15th ed., Washington, D.C.

Barbora, A.C., S.C. Borah, and D. Bhattacharya. 2002. Effect of weed management on yield and quality of Khasi mandarin (Citrus reticulata Blanco). Indian J. Citricult. 1:62-65.

Bond, W. and A.C. Grundy. 2001. Non-chemical weed management in organic farming systems. Weed Res. 41:383-405.

Bredell, G.S. 1973. Response of citrus trees to plastic mulching. Proc. Intl. Soc. Citricult. Murcia-Valencia, Spain, p. 387-394.

Daar, S. 1986. Update: Suppressing weeds with allelopathic mulches. The IPM Practitioner. April. p. 1-4, Thompson Publ., Fresno, CA.

Donadio, L.C., J.R.M. Cabrita, A.R.S. Jose, and D.A. Banzatto. 1988. Cultural practices for citrus orchard formation. Proc. Sixth Intl. Citrus Congr. Tel Aviv, Israel, 1988. p. 615-619.

Doug, A.D., L.A. Randy, E.B. Robert, and M. Bruce. 2002. Weed dynamics and management strategies for cropping systems in the northern Great Plains. Agron. J. 94:174-185.
Eissa, M.A. and B.M. Helail. 1997. A comparative study on some weed control treatments in Balady lime nursery. Ann. Agr. Sci. Moshtohor (Egypt) 35:935-947.

Faber, B.A., A.J. Downer, and J.A. Menge. 2001. Differential effects of mulch on citrus and avocado. ISHS Acta Hort. 557: VII Intl. Symp. Orchard Plantation Syst., 30 July 2001. Vol. 1. No. 63. Nelson, New Zealand.

Frans, R., R. Talbert, and D. Marx. 1986. Experimental design and techniques for measuring and analyzing plant responses to weed control practices, p. 29-46. In: N.D. Camper (ed.). Research methods in weed science. Champaign, IL.

Ghosh, P.K., Devi Dayal, K.K. Bandyopadhyay, and M. Mohanty. 2006. Evaluation of straw and polythene mulch for enhancing productivity of irrigated summer groundnut. Field Crop Res. 99:76-86.

Gleason, M., S. Wegulo, and G. Nonnecke. 2001. Efficacy of straw mulch for suppression of anthracnose on day-neutral strawberries. ISU Ext. FG-601:48. Iowa State Univ., Ames, IA.

Goff, W.D., M.G. Patterson, and M.S. West. 1991. Orchard floor management practices influence elemental concentrations in young pecan trees. HortScience 26:1379-1381.

Hoyt, G.D. and W.L. Hargrove. 1986. Legume cover crops for improving crop and soil management in the southern United States. HortScience 23:397-402.

Hussein, H.F. and S.M.A. Radwan. 2002. Bioorganic fertilization of potato under plastic mulches in relation to quality of production and associated weeds. Arab Univ. J. Agr. Sci., Ain Shams Univ. Cairo (Egypt) 10:287-309.

Kalita, H. and R.K. Bhattacharyya. 1995. Integrated weed management in Assam lemon in lemon (Citrus limon Burm.f.) orchards. Hort. J. 8:95-100.

Li, Y., Z. Sun, X. Zhuang, L. Xu, S. Chen, and M. Li. 2003. Research progress on microbial herbicides (Review). Crop Prot. 22:247-252.

Ligneau, L.M. and T.A. Watt. 1995. The effects of domestic compost upon the germination and emergence of barley and six arable weeds. Ann. Appl. Biol. 126:153-162.

Lima, A., A. De, R.C. Caldas, A.L. Borges, C.H.S.P. Ritzinger, A.V. Trindale, M. De, M. Pires, M.M.B.C. Midlej, H.T.D.A.C. Mata, and J.D.A.S. Souza. 2002. Intercropping and weed control in yellow passion fruit orchards. Revista Brasileira de Fruticultura 24:711-713.

MacRae, A.W., W.E. Mitchem, D.W. Monks, M.L. Parker, and R.K. Galloway. 2007. Tree growth, fruit size, and yield response of mature peach to weed-free intervals. Weed Technol. 21:102105

Martini, G., A.F.A. Pedrinho, Jr., G.V. Felici, F.M. Piva, and J.C. Durggan. 2002. Efficacy of a new formulation of glyphosate for control of bermuda grass (Cynodon dactylon) in citrus orchards. Revista Brasileira de Fruticultura 24:683-686.

Merwin, I.A. 1995. IPM systems for orchard soils: Groundcover management versus weed control. Proc. New Engl Fruit Mtg., Sturbridge, MA. Jan. 1995. 101:43-49.

Mohanty, S., R.K. Sonkar, and R.A. Marathe. 2002. Effect of mulching on Nagpur mandarin cultivation in drought prone region of Central India. Indian J. Soil Conserv. 30:286-289.

Mukhopadhyay, S.K. 2006. Paradigms in weed management. National Symposium on Conservation Agriculture and Environment, 26-29 Oct. 2006. BHO. Varanasi. p. 295-296.
Pedersen, H.L. 1999. Alternative to herbicide use for controlling weeds in apple orchards. Rivista di Frutticollture e di Ortofloricplturo 61:81-83.

Radosevich, S., J. Holt, and C. Ghersa. 1997. Weed ecology: Implications for management. 2nd ed. Wiley, New York.

Radwan, S.M.A. and H.F. Hussein. 2001. Response of onion (Allium cepa, L.) plants and associated weeds to biofertilization under some plant mulched. Ann. Agr. Sci., Ain Shams Univ. Cairo (Egypt) 46:543-564.

Robinson, D.W. 1988. Mulches and herbicides in ornamental plantings. HortScience 23:547552.

Sabbah, S.M., A.T. Assal, and E.A. Ghaly. 1994. The relative influence of some herbicides (trifluralin, glyphosate, and bromacil) on yield, fruit quality, and leaf mineral composition of Washington navel orange trees. Ann. Agr. Sci. (Egypt) 39:721-729.

Santinoni, L.A. and N.A. Silva. 1995. Growth, yield, and maturation of common mandarin under different soil management practices. Horticultura Argenteina 14:5-11 (Weed Abst 1996, 45:3233).

Shirgure, P.S., R.K. Sonkar, S. Shyam, and P. Panigrah. 2003. Effect of different mulches on soil moisture conservation, weed reduction, growth and yield of drip irrigated Nagpur mandarin (Citrus reticulata). Indian J. Agr. Sci. 73:148-152.

Singh, A., M. Singh, D.V. Singh, and M. Singh 1985. Relative efficacy of organic mulch and herbicides for weed control in Cymbopogon species. Ann. Conf. Indian Soc. Weed Sci., p. 77 (Abstract).

Singh, M. and S. Singh. 2003. Response of some important citrus weeds to two formulations of glyphosate applied at the three growth stages. The BCPC Intl. Congr. Crop Sci. Technol. p. 1053-1058.

Singh, S. and M. Singh. 2004. Effect of growth stage on trifloxysulfuron and glyphosate efficacy in twelve weed species of citrus groves. Weed Technol. 18:1031-1036.

Teasdale, J.R. and C.L. Mohler. 1993. Light transmittance, soil temperature, and soil moisture under residue of hairy vetch and rye. Agron. J. 85:673-680.

Timossi, P.C., P.L. Da, and C.A. Alves. 2001 Effects of clomoazone drift simulation on Hamlin orange fruits. Revista Brasileria de Fruiticultura 23:310-314

Timothy, K.B. 2007. Effects of mulch type and fertilizer placement on weed growth and soil $\mathrm{pH}$ and nutrient content. HortTechnology 17:174-177.

Tucker, D.P.H. and M. Singh. 1984. Florida citrus weed management, p. 12-31. In: J.L. Knapp (ed.). Florida Citrus integrated pest management handbook. Florida Coop. Ext. Serv., University of Florida, Gainesville.

Tworkoski, T.J. and D.M. Glenn. 2001. Yield, shoot and root growth, and physiological responses of mature peach trees to grass competition. HortScience 36:1214-1218.

Wehtje, G. and R.H. Walker. 1997. Interaction of glyphosate and 2,4-DB for the control of selected morningglory (Ipomoea spp.) species. Weed Technol. 11:152-156.

Weibel, F.P. and U. Niggli. 1990. Weed control with organic mulches in apple orchards: Effects on weed growth, dynamics of nitrogen in soil solution and on microbial respiration in soil, v20, p. 147-163. In: Proc. Third Intl. Conf. IFOAM, Nonchemical Weed Control, Linz, Austria. 